Reprod. Nutr. Dévelop., 1981, 21 (6A), 953-960.

\title{
La régression de l'intestin caudal chez l'embryon de poulet. Stade de détermination des dégénérescences cellulaires et rôle du mésenchyme associé
}

\author{
par R. LANOT, Anne-Marie BAUTZ
}

Laboratoire de Zoologie générale, Université de Nancy I, Faculté des Sciences, C.O. no 140, 54037 Nancy cedex.

Summary. Regression of chick embryo tail gut. Stage of defermination of cell degeneration and role of the associated mesenchyme.

In chick embryo the complete degeneration of the tail gut is carried out between $\mathrm{H}$ and $\mathrm{H}$ stages 20 to 24 and cell alterations are noticeable from stage 19. This paper reports a study to ascertain whether degeneration of the tail gut cells is already determined.

The tail guts of donor embryos were taken at stages 17 to 20 and implanted into the leg bud of 4 to 5-day host embryos. In another series, stage 19 tail guts were implanted with their associated mesenchyme.

The fate of the implants was histologically studied in the hosts at different times during their subsequent development.

The younger grafts formed tubular structures almost identical to the normal tail gut. Implanted stage 19 tail guts with practically no mesenchyme did not degenerate; they maintained their structure but did not differentiate further. The tail guts implanted with associated mesenchyme degenerated, and stage 20 tail guts also regressed.

The death of the tail gut cells was not determined until the degeneration processes had actually begun, the fate of the tail gut cells depending on their environment.

\section{Introduction.}

Chez l'embryon de Poulet l'intestin caudal dégénère au cours des stades 20 à 24 , entre trois jours et demi et quatre jours et demi d'incubation (Boyden, 1922). Des altérations cellulaires s'observent déjà trois heures plus tôt, au stade 19, dans l'épithélium intestinal caudal (Lanot, 1980). Cette observation pourrait signifier que la régression de cet épithélium est déterminée dès ce stade. C'est à la vérification de cette hypothèse que s'attache d'abord le présent travail. Il vise en outre à mettre en évidence le rôle des tissus avoisinants dans la détermination des dégénérescences cellulaires. 


\section{Matériel et méthodes.}

Il s'agit d'étudier l'évolution de l'intestin caudal d'embryons de Poulet pris à différents stades lorsqu'il est transplanté dans une situation différente, isolément ou en association avec le mésenchyme du bourgeon caudal. Pour ce faire l'intestin caudal est prélevé par dissection (fig. 1) sur des embryons de Poulet des stades 17 à 20 (30 à 40 paires de somites, 60 d̀ 75 h d'incubation). Auparavant les embryons subissent un traitement de courte durée (moins de $10 \mathrm{~min}$ ), à la température du laboratoire par une solution de trypsine à 0,3 p. 100 dans le liquide de Moscona (Tyrode sans calcium ni
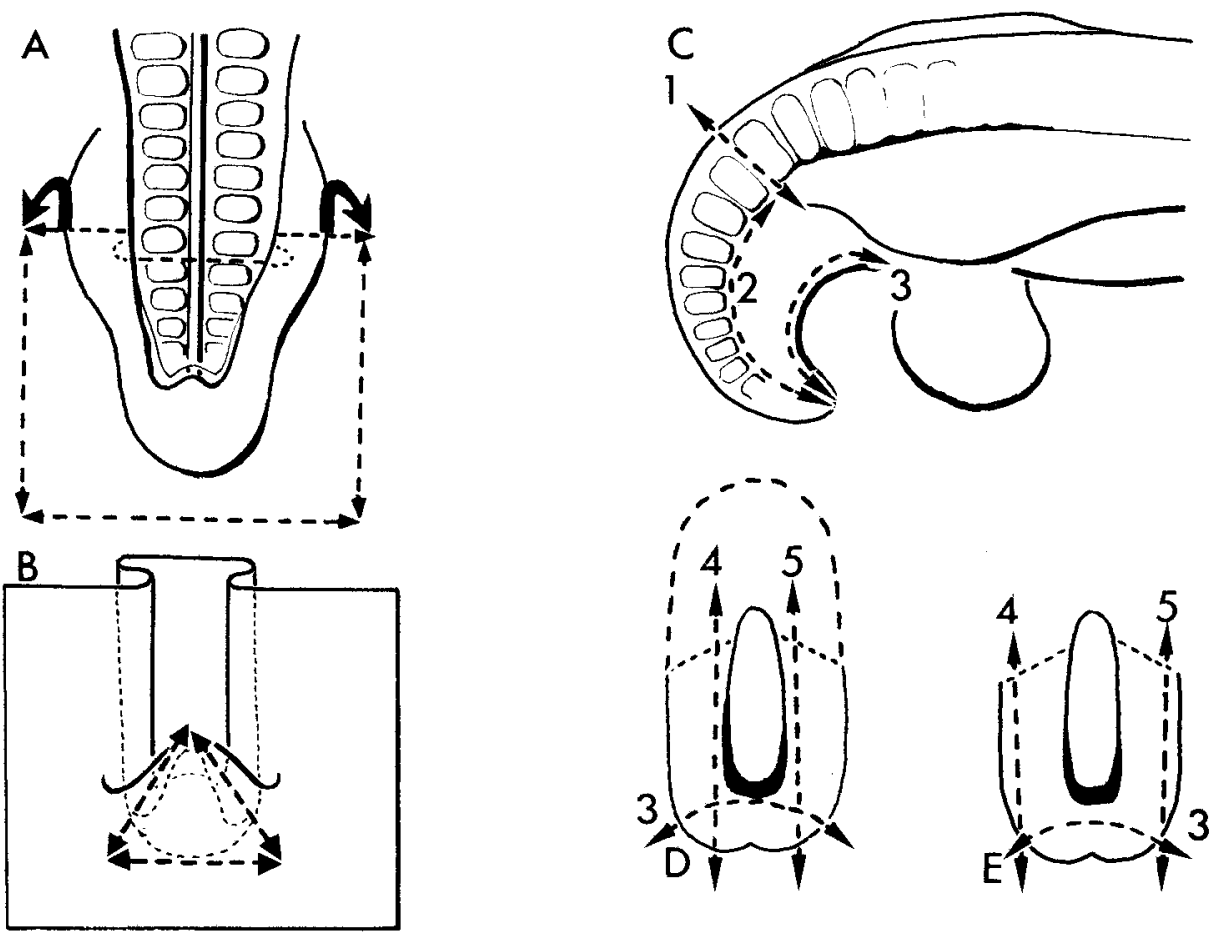

FIGURE 1

A) Schéma montrant la méthode d'isolement de l'épithélium intestinal aux sfades 17 et 18 : une plage d'endoderme débordant largement sur la partie caudale de l'aire pellucide est découpée puis décollée.

B) Prélèvement de l'intestin caudal à partir de la plage d'endoderme isolé.

C) Schéma montrant la méthode d'isolement et de prélèvement des transplants plus âgés (stades 19 ef 20). Des sections longitudinales passant à la base des somites permettent de dégager la partie dorsale du fube intestinal. La ligne primitive est extirpée aisément. Le reste du mésenchyme est éliminé (D) pour l'essentiel par des sections dorso-ventrales longiłudinales. Dans le cas où ce mésenchyme est conservé (E) seul l'ectoderme du bourgeon caudal est enlevé. Tant que le fube intestinal n'est pas prêt pour la transplantation il est nécessaire de le conserver attaché à l'embryon. II n'est pas possible de réaliser ces opérations sur un bourgeon caudal isolé. 
magnésium), dans laquelle on pratique ensuite la dissection. La préparation de l'ébauche intestinale aux stades 17 et 18 , faite par arrachage très progressif de l'endoderme à partir de l'aire pellucide, fournit exclusivement de l'endoderme au niveau du bourgeon caudal. Celle faite aux stades 19 et 20 laisse subsister au pire quelques dizaines de cellules du mésenchyme (les contrôles microscopiques ont été effectués après coloration au rouge neutre sur les dissections les moins réussies ou sur celles donf la durée, supérieure à $20 \mathrm{~min}$, nous avait parue excessive).

Le lambeau d'endoderme, dans lequel est incluse l'ébauche intestinale aux stades 17 ef 18, est transféré rapidement dans frois bains successifs de Tyrode, puis il est étalé sur un fond noir ef l'ébauche intestinale est découpée. Les infestins caudaux prélevés aux stades 19 et 20 sont aspirés deux ou trois fois à la pipette dans la solution de trypsine. lls sont ensuite transférés dans trois bains successifs de Tyrode où ils subissent la même agitation. Celle-ci a pour effet de les débarrasser de la plupart des cellules mésenchymateuses ; il en subsiste néanmoins quelques-unes. Ce processus de rinçage supprime aussi les cellules endodermiques nécrosées lorsqu'il en existe au stade 20. L'intestin est ensuite injecté, au moyen d'une pipette de verre à bords tranchants, à la base du bourgeon de patte d'embryons de 4 à 5 jours d'incubation (stades 22 à 25). Afin d'éviter l'expulsion du transplant (celle-ci survient, le cas échéant, dans la demiheure qui suit), une cavité est pratiquée préalablement dans le bourgeon de patte en aspirant un peu de mésenchyme. Les receveurs qui expulsent le transplant ne sont pas retenus. Ceux qui le conservent sont fixés 12 à $72 \mathrm{~h}$ plus tard selon l'âge des transplants, puis analysés histologiquement.

\section{Résultats.}

A. Intestins prélevés aux stades 17 et 18. - A ces stades l'intestin caudal commence seulement à se modeler. Lorsqu'il est transplanté l'épithélium intestinal continue sa morphogenèse, mais celle-ci n'est jamais parfaite. Dans les sept cas retenus il constitue finalement un tube dont la paroi est d'épaisseur uniforme. Dans l'embryon normal, au contraire, on observe des différences d'épaisseur importantes dans la moitié antérieure de l'intestin caudal. Cependant la structure histologique du tube formé par les transplants est normale (fig. 2D et E). Elle correspond à celle de la moitié postérieure de l'intestin caudal aux słades 19 ef 20 . Ces tubes intestinaux atypiques se sont formés dans le tissu musculaire ou le derme de la patte en voie de différentiation.

B. Transplants prélevés au stade 19. - Dans l'embryon normal, l'intestin caudal, au stade 19 atteint son plus grand développement; mais ainsi que nous l'avons rappelé plus haut des altérations minimes sont déjà décelables dans certaines cellules intestinales. Deux types d'expériences ont été réalisés à ce stade.

1. Intestins greffés avec quelques cellules de leur mésenchyme homologue. - Ces intestins greffés se maintiennent vivants dans tous les cas (7). lls conservent leur organisation histologique, mais ils ne progressent pas dans leur différenciation (fig. $2 A, B$ et $C$ ). Les transplants se comportent de façon identique quel que soit le type de différenciation des tissus hétérologues qui les entourent : mésenchyme du derme, muscle strié ou cartilage (fig. 2B, $C$ et $D$ ). Ces tissus eux-mêmes ne paraissent pas avoir été influencés par les greffons. 

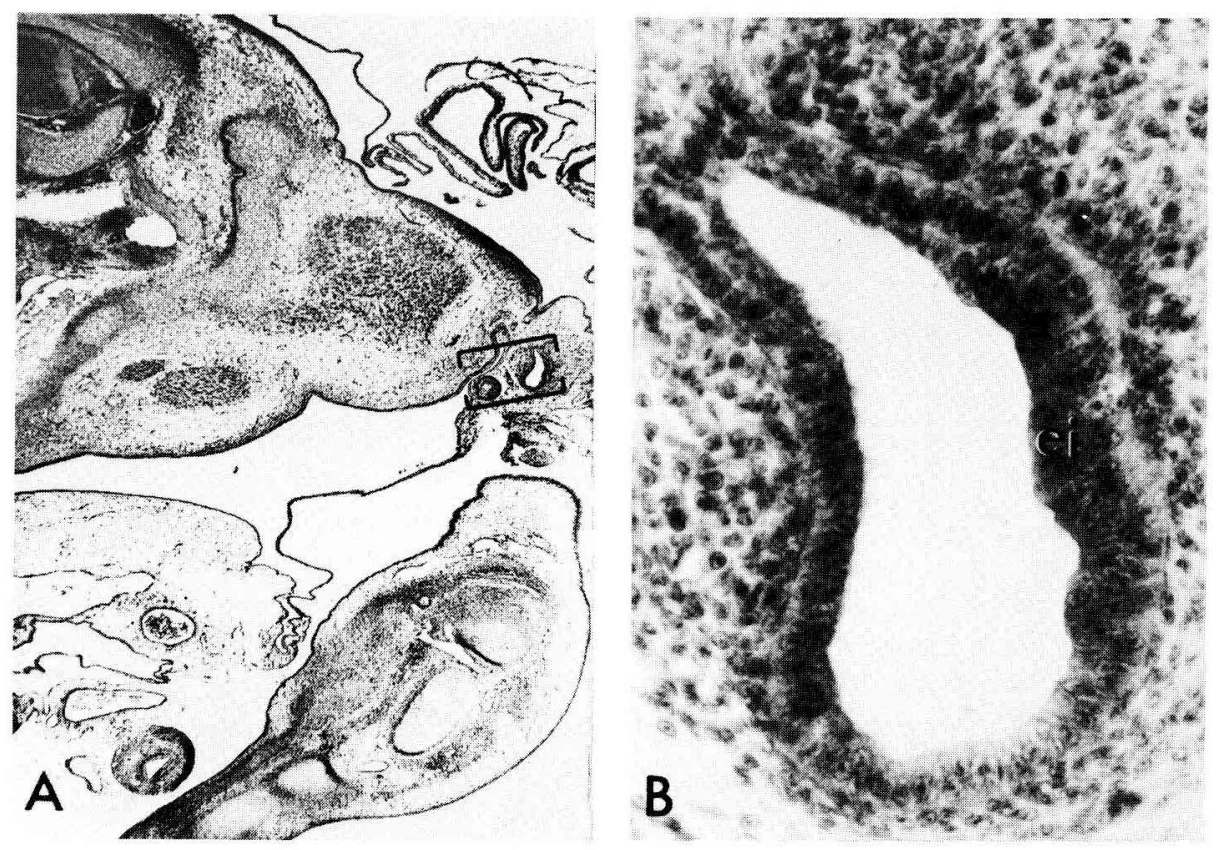

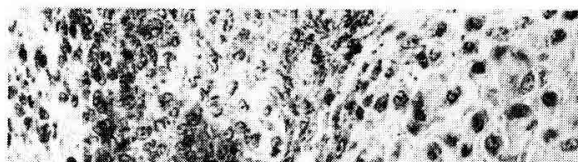

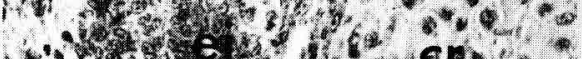

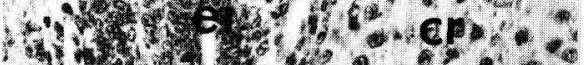

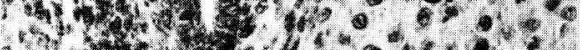

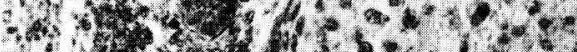

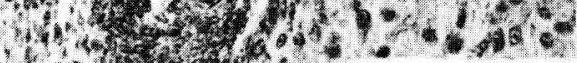
(4)
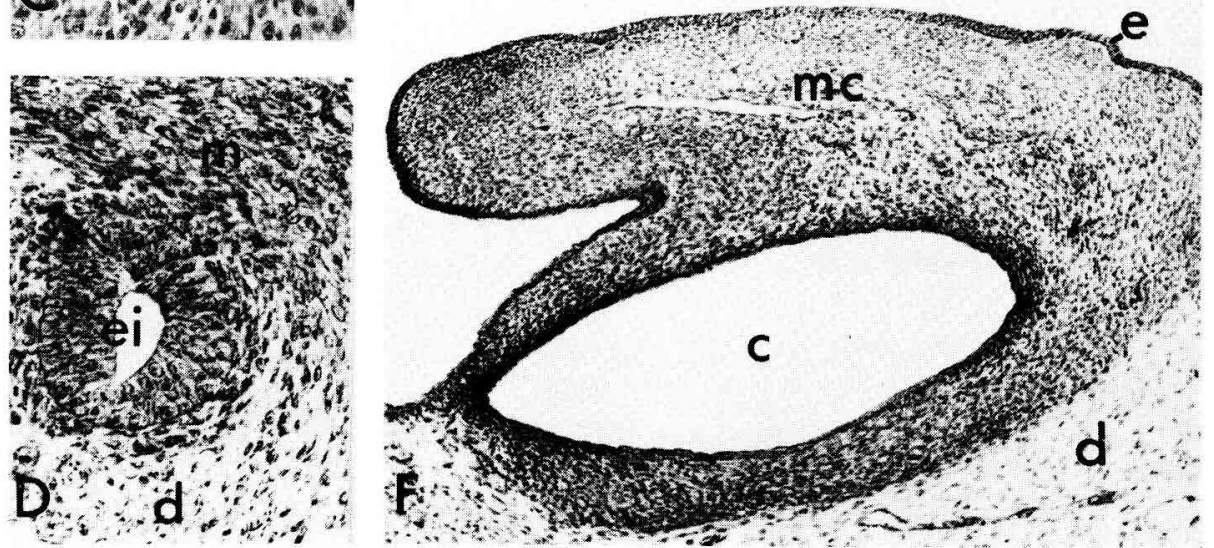
2. Infestins greffés avec leur mésenchyme homologue. - Lorsque les intestins sont implantés avec le mésenchyme latéral qui leur est associé (4 cas), ils dégénèrent complètement (fig. 2F).

C. Intestins prélevés au stade 20. - Au moment de leur prélèvement les tubes intestinaux onf déjà commencé à dégénérer dans leur moitié antérieure. Cette région devenue très fragile est souvent perdue au cours des manipulations successives du transplant, si bien que celui-ci n'est formé en général (5 cas sur 7) que de la moifiè postérieure du tube intestinal, ef comme précédemment de quelques cellules du mésenchyme. Dans tous les cas les transplants dégénèrent et sont éliminés en $24 \mathrm{~h}$ (fig. 3).

\section{Discussion.}

La transplantation d'épithélium intestinal caudal isolé permet de préciser le stade à partir duquel la dégénérescence des cellules est déterminée. Ainsi l'épithélium intestinal prélevé au stade 19 et greffé, seul ou avec très peu de cellules mésodermiques associées, dans l'ébauche de patte, se maintient en vie au lieu de dégénérer. Les altérations cellulaires présentes à ce stade ne sont donc pas irréversibles. Au contraire la même expérience, lorsqu'elle est réalisée au stade 20 (soił environ $3 \mathrm{~h}$ plus tard),

\section{FIGURE 2}

A) Aspect histologique d'un porte-greffe contenant un transplant prélevé au stade 19 (LJ) et fixé 48 h oprès son implantation. Maintien de l'épithélium intestinal ( $\times$ 47).

B) Aspect à plus fort grossissement de cet épithélium. $(\times 750)$.

C) Epithélium infestinal (ei) prélevé au stade 19 ef fransplanté dans un terrifoire chondrogène $(\times 470)$.

D) Epithélium intestinal (ei) prélevé au stade 18 et transplanté à la limife du derme (d) et du territoire musculaire $(m)$ de la patte $(\times 470)$.

E) Aspect hisfologique d'un tel transplant. La structure de l'épithélium équivaut à celle d'un intestin caudal normal au stade 19. Les expansions (*) situées au-dessus du bord apical des cellules (ligne sombre marquée par les flèches correspondent chez l'embryon normal à des vésicules de pinocytose) $(\times 1875)$.

F) Aspect histologique d'un transplant prélevé au stade 19 comprenant l'épithélium intestinal et le mésenchyme associés. Le transplant a été implanté sous l'épiderme (e). Seul le mésenchyme caudal $(\mathrm{mC})$ subsiste, il se distingue aisément du derme de l'hôte. L'épithélium intestinal a dégénéré, à sa place existe encore une cavité (c), $48 \mathrm{~h}$ après la transplantation. Le pourtour de la cavité est bordé par des cellules du mésenchyme plus tassées dont la structure est la même que celle montrée dans la figure 3D $(\times 187)$. 

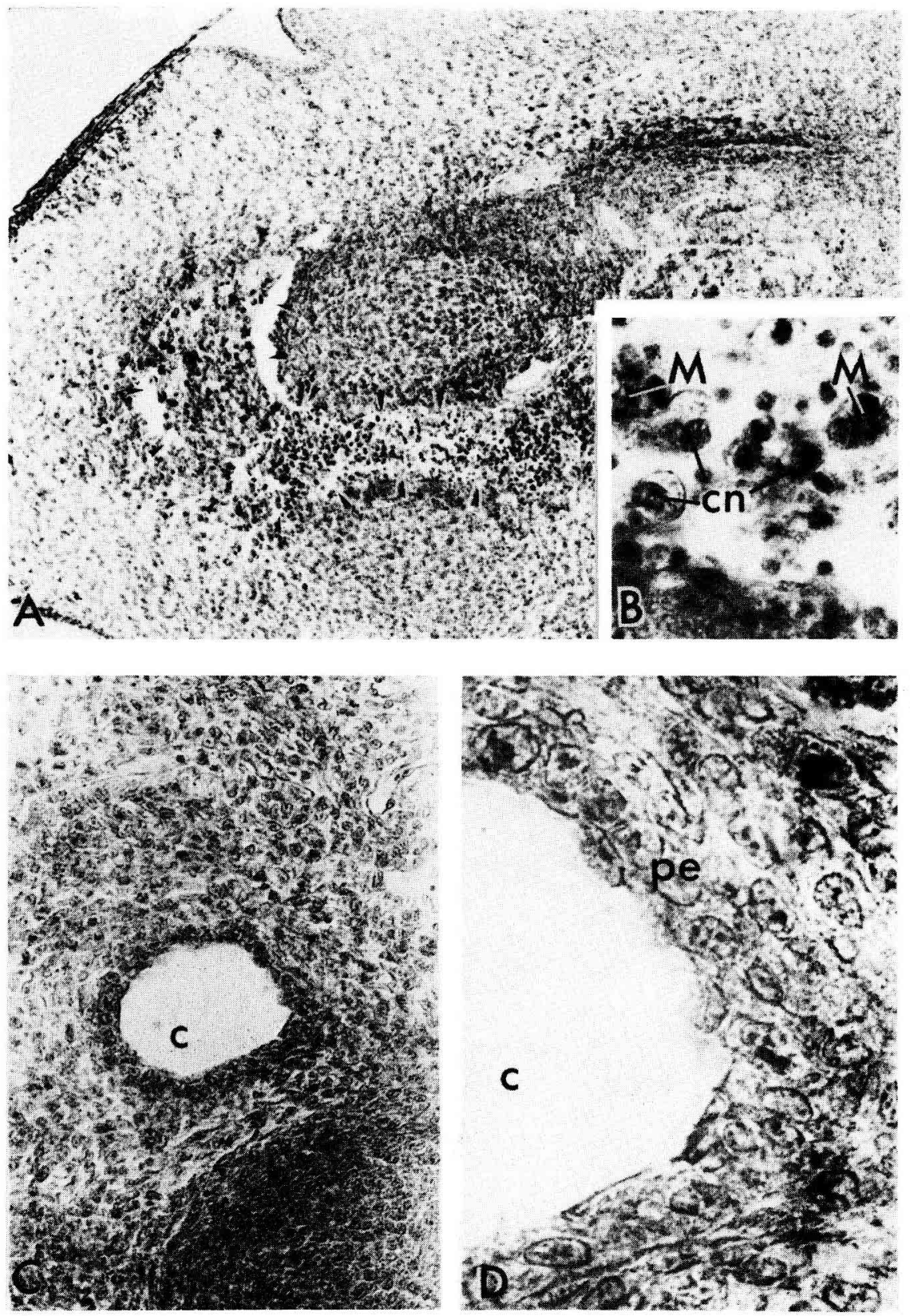
aboutit à la régression complète de l'intestin transplanté : la dégénérescence de toutes les cellules intestinales esł devenue irréversible. La transplantation de l'épithélium intestinal en association avec son mésenchyme homologue permet de localiser les facteurs qui induisent cette dégénérescence. En effet, lorsque le mésenchyme latéral du bourgeon de queue existe en quantité importante, dans les transplants prélevés au stade 19, ef qu'il recouvre régulièrement l'épithélium intesłinal, celui-ci dégénère. II se maintient lorsque, la quantité de mésenchyme homologue étant réduite au minimum possible, il entre en contact avec le mésenchyme hétérologue. De toute évidence le mésenchyme latéral du bourgeon de queue n'est plus favorable au maintien de l'épithélium intestinal, à partir du stade 19 alors que le mésenchyme du bourgeon de patte et les tissus qui en dérivent le sont. Une telle constatation implique que la dégénérescence du bourgeon caudal dépend de facteurs résidant dans son environnement. Mais elle ne renseigne pas sur la nature des facteurs en cause, ni sur le fait de savoir si c'est l'environnemenł qui s'altère ou si c'est l'épithélium qui se modifie. Elle permet cependant de rejeter l'hypothèse, formulée par Boyden, selon laquelle le reliquat de ligne primitive incorporé au bourgeon caudal aurait un rôle déterminant dans l'involution de l'intestin caudal, puisque ce phénomène se réalise dans les transplants dépourvus de ligne primitive. Zwilling (1946) avait d'ailleurs montré que la suppression de la ligne primitive en arrière du bouton terminal, avant la formation du bourgeon de queue, est sans conséquence sur l'évolution de cette structure. Selon ce même auteur (Zwilling, 1952 et 1956) les phénomènes de réduction qui se déroulent dans le bourgeon caudal des embryons normaux ou « rumpless » résultent du contrôle génétique plus ou moins éfendu de zones potentiellement nécrogènes. Une telle conception pourrait laisser penser que les dégénérescences génétiquement programmées sont l'aboutissement d'un processus rigoureusement automatique limité pour l'essentiel aux cellules qui dégénèrent. En réalité, nous avons constaté que leur environnement est aussi impliqué dans ce processus.

\section{FIGURE 3}

A) Epithélium intestinal prélevé au stade 20 ef fixé $12 \mathrm{~h}$ plus tard. Il a entièrement dégénéré, les limites sont indiquées par des flèches. Les débris sont phagocytés par des macrophages issus vraisemblablement du mésenchyme voisin $(\times 187)$.

B) Détail de la zone nécrotique. $\mathrm{cn}=$ cellules nécrotiques; $M=$ macrophages ( $\times 1875$ ).

C) Epithélium intestinal prélevé au stade 20 et fixé 48 h plus tard. L'épithélium a entièrement dégénéré, à sa place subsiste une cavité $(\times 470)$.

D) Détail a plus fort grossissement de la limife mésenchymateuse (pe = pseudoépithélium) de cetfe cavifé $(\times 1875)$. 
L'évolution de l'épithélium intestinal caudal semble pouvoir s'expliquer, comme dans les autres secteurs du tube digestif (Sigot, 1962, 1963 ; Le Douarin, 1964 ; Soriano et al., 1964, 1965), par le jeu des interactions qui s'exercent entre l'épithélium digestif ef son mésenchyme homologue.

Reçu en jonvier 1981. Accepté en mai 1981.

\section{Références}

BOYDEN E. A., 1922. The development of the cloaca in birds, with special reference to the origin of the bursa of Fabricius, the formation of urodeal sinus, and the regular occurence of cloacal fenestra. Am. J. Anat., 30, 163-201.

HAMBURGER V., HAMILTON H. L., 1951. A series of normal stages in the development of the chick embryo. J. Morph., 88, 49-92.

LANOT R., 1980. Régression de l'intestin caudal et phénomènes associés chez l'embryon de poulet. Arch. Anat. micr. Morph. exp., 69, 243-257.

LE DOUARIN N., 1964. Etude expérimentale de l'organogenèse du tube digestif et du foie chez l'embryon de poulet. Bull. biol. Fr. Belg., 98, 544-676.

SIGOT M., 1962. Sur le rôle du mésenchyme dans la différenciation des glandes du proventricule chez le poulet. C. R. Acad. Sc. Paris, sér. D, 254, 2439-2441.

SIGOT M., 1963. Induction de la formation de glycogène dans l'épithélium du proventricule par le mésenchyme du gésier chez l'embryon de poulet. C. R. Acad. Sc. Poris, sér. D, 256, 4970-4971.

SORIANO L., SAXEN L., VAINIO T., TOIVONEN S., 1964. The development of the oesophagal and tracheobranchial epithelia in vitro. Acfa anat., 57, 105-115.

SORIANO L., 1965. Différenciation des épithéliums du tube digestif in vitro. J. Embryol. exp. Morph., 14, 119-128.

ZWILLING E., 1942. The development of dominant rumplessness in chick embryos. Genetics, 27, 641656.

ZWILLING E., 1946. Regulation in the chick allantois. J. exp. Zool., 101, 445-453.

ZWILLING E., 1956. Teratogenesis. In WILLIER B. H., WEISS P. A., HAMBURGER V., Analysis of development. Saunders ed. Philadelphia and London. 